AEI-2002-070

CERN-TH/2002-232

LAPTH-935/02

hep-th/0209103

\title{
A note on the perturbative properties of BPS operators
}

\author{
Gleb Arutyunov ${ }^{*, * *}$, Emery Sokatchev ${ }^{\ddagger, \ddagger \ddagger}$ 円 \\ * Max-Planck-Institut für Gravitationsphysik, Albert-Einstein-Institut, \\ Am Mühlenberg 1, D-14476 Golm, Germany \\ $\ddagger$ CERN Theoretical Division, CH-1211 Geneva 23, Switzerland \\ ¥† Laboratoire d'Annecy-le-Vieux de Physique Théorique LAPTH \\ BP 110, F-74941 Annecy-le-Vieux Cedex, France
}

\begin{abstract}
We discuss the perturbative behavior of the $1 / 2$ BPS operators in $\mathcal{N}=2$ SCFT on the example of two very similar quadrilinear composite operators made out of hypermultiplets. An explicit one-loop computation shows that one of them is protected while the other acquires an anomalous dimension. Although both operators are superconformal primaries in the free case, the quantum corrections make the latter become a 1/2 BPS descendant of the Konishi multiplet, while the former remains primary. The comparative study of these two operators at higher orders may be helpful in understanding the quantum properties of the Konishi multiplet.
\end{abstract}

\footnotetext{
${ }^{1}$ email:agleb@aei-potsdam.mpg.de

2 email:Emery. Sokatchev@cern.ch

** On leave of absence from Steklov Mathematical Institute, Gubkin str.8, 117966, Moscow, Russia

${ }^{3}$ UMR 5108 associée à l'Université de Savoie
} 


\section{Introduction}

A lot of progress in understanding the classical and quantum properties of the superconformal field theories has been achieved since the discovery of the AdS/CFT correspondence. This includes both the development of the abstract superconformal representation theory as well as the analysis of the correlation functions of concrete composite operators obtained in different regimes of the correspondence.

A special class of composite operators in $\mathcal{N}=4 \mathrm{SYM}$ playing a privileged rôle in the AdS/CFT dictionary are the so-called BPS short operators, i.e. operators annihilated by a certain fraction of the Poincaré supercharges $Q$. They form isolated series of representations of the superconformal group with quantized (protected) conformal dimension (for a full classification see, e.g., [1]).

It is important to realize that the symmetry protects such BPS operators only if they are superconformal primary, i.e., if they are annihilated by all the generators $S$ of special conformal supersymmetry. For a given composite operator in $\mathcal{N}=4 \mathrm{SYM}$ the latter property is not obvious. It may happen that an operator looks BPS short (it is annihilated by some charges $Q$ ), but in fact it is a superconformal descendant of another, long multiplet. At the same time, such an operator may still be a conformal primary (i.e., it is annihilated by the conformal boosts) pure state and may have well-defined but unprotected conformal dimension. The issue is rather subtle because the superconformal "primarity" is lost only at the quantum level. This possibility was first pointed out in [2] in the $\mathcal{N}=1$ framework and has since been reiterated many times (see, e.g., [3]).

The fact that a long supermultiplet lying on the threshold of the unitary bound in the classical theory is a reducible representation of the superconformal group has been known for a long time (see, e.g., [1]). Recently, a detailed analysis of the decomposition of long multiplets into semishort or BPS short multiplets has been carried out in [4]. In particular, when the superconformal primary long multiplet of the $\mathcal{N}=4(\mathcal{N}=2)$ algebra is a Lorentz scalar, one finds $1 / 4(1 / 2)$ BPS multiplets in its decomposition.

In this note we present a concrete and simple example of a "fake" and a "true" $1 / 2$ BPS operators in $\mathcal{N}=2$ SCFT and discuss their nature in perturbation theory. After recalling the necessary tools to define the Konishi superfield in the framework of the $\mathcal{N}=2$ superspace in Section 2, in Section 3 we extract from it a superconformal descendant (but a conformal primary) having the quantum numbers and the appearance of an $\mathcal{N}=21 / 2 \mathrm{BPS}$ operator. It is obtained by acting on the Konishi superfield with four (i.e., half of the total number) spinor derivatives and by using the interacting field equations for the elementary constituents. This operator is a single-trace quadrilinear combination of the elementary hypermultiplet (HM) superfields and involves color group commutators. At the same time, we construct another, very similar composite with fully symmetrized color indices which is supposed to be a true protected 1/2 BPS primary operator. Our present understanding of the short composite operators does not allow us to make definitive statements about the latter, we can only rely on perturbative evidence [7]. In Section 4 we examine the one-loop corrections to the two-point functions of both operators and show explicitly the origin of

\footnotetext{
${ }^{1}$ It was precisely the presence of a commutator term in the definition of a composite chiral operator that was interpreted in 2] as a signal that it may be a descendant of some lower dimensional non-chiral operator. For relatively low conformal dimensions this criterium for identifying descendant operators in perturbation theory was used, e.g. in $[$, 国].
} 
the anomalous dimension of the former. Since this is precisely the anomalous dimension of the "parent" Konishi multiplet, understanding the mechanism which distinguishes the "fake" $1 / 2$ BPS operator from the very similar "true" one at higher orders in perturbation theory may prove useful in an attempt to compute the exact anomalous dimension of the Konishi multiplet. In addition, the example that we consider in the paper can serve as a concrete and simple illustration of the general representation theory discussion in Ref. 何. Finally, in Section 5 we formulate several open questions, the main one being: How to test the "primarity" of a given operator?

\section{$2 \mathcal{N}=4 \mathrm{SYM}$ in terms of $\mathcal{N}=2$ superfields}

The two $\mathcal{N}=2$ ingredients of the $\mathcal{N}=4 \mathrm{SYM}$ theory are the HM and the $\mathcal{N}=2 \mathrm{SYM}$ multiplet. In harmonic superspace [8] the former is described by a Grassmann (G-)analytid] (also frequently called " $1 / 2$ BPS") superfield of $U(1)$ charge +1 ,

$$
D_{\alpha}^{+} q^{+}=\bar{D}_{\dot{\alpha}}^{+} q^{+}=0 \Rightarrow q^{+}=q^{+}\left(x, \theta^{+}, \bar{\theta}^{+}, u\right) .
$$

Here $D_{\alpha, \dot{\alpha}}^{+}=u_{i}^{+} D_{\alpha, \dot{\alpha}}^{i}$ and $u_{i}^{ \pm}, i=1,2$ form an $S U(2)$ matrix of harmonic variables on the two-sphere $S^{2} \sim S U(2) / U(1)$. In the free case the HM satisfies the equation of motion

$$
D^{++} q^{+}=0,
$$

which takes the form of a harmonic (H-)analyticity condition. Here

$$
D^{++}=\partial_{u}^{++}-2 i \theta^{+} \sigma^{\mu} \bar{\theta}^{+} \partial_{\mu}
$$

is the supercovariant harmonic derivative in the G-analytic basis, which also plays the rôle of the raising operator of $S U(2)$. The solution of eq. (2) is the ultrashort on-shell superfield

$$
q^{+}=q^{i}(x) u_{i}^{+}+\theta^{+\alpha} \psi_{\alpha}(x)+\bar{\theta}_{\dot{\alpha}}^{+} \bar{\kappa}^{\dot{\alpha}}(x)+2 i \theta^{+} \sigma^{\mu} \bar{\theta}^{+} \partial_{\mu} f^{i}(x) u_{i}^{-},
$$

where the physical components of the $\mathcal{N}=2 \mathrm{HM}$, the doublet of scalar fields $q^{i}(x)$ and the fermions $\psi, \bar{\kappa}$, satisfy their free field equations

$$
\square q^{i}(x)=\not \partial \psi=\not \partial \bar{\kappa}=0 .
$$

The $\mathcal{N}=2 \mathrm{SYM}$ multiplet is described by another G-analytic superfield, this time of $U(1)$ charge $+2, V^{++}\left(x, \theta^{+}, \bar{\theta}^{+}, u\right)$. It plays the rôle of the gauge connection in the covariantized harmonic derivative

$$
\nabla^{++}=D^{++}+i g V^{++}
$$

so that the flat commutation relations with $D_{\alpha, \dot{\alpha}}^{+}$are preserved:

$$
\left[\nabla^{++}, D_{\alpha, \dot{\alpha}}^{+}\right]=0
$$

\footnotetext{
${ }^{2}$ Grassmann analyticity is the natural generalization of the notion of chirality in the case of extended supersymmetry.
} 
In the Wess-Zumino gauge the component content of $V^{++}$is reduced to the off-shell $\mathcal{N}=2$ gauge multiplet:

$$
\begin{aligned}
V_{\mathrm{WZ}}^{++}= & -2 i \theta^{+} \sigma^{\mu} \bar{\theta}^{+} A_{\mu}(x)-i \sqrt{2}\left(\theta^{+}\right)^{2} \bar{\phi}(x)+i \sqrt{2}\left(\bar{\theta}^{+}\right)^{2} \phi(x) \\
& +4\left(\bar{\theta}^{+}\right)^{2} \theta^{+\alpha} \psi_{\alpha}^{i}(x) u_{i}^{-}-4\left(\theta^{+}\right)^{2} \bar{\theta}_{\dot{\alpha}}^{+} \bar{\psi}^{\dot{\alpha} i}(x) u_{i}^{-} \\
& +3\left(\theta^{+}\right)^{2}\left(\bar{\theta}^{+}\right)^{2} D^{i j}(x) u_{i}^{-} u_{j}^{-}
\end{aligned}
$$

In the $\mathcal{N}=4$ theory the $\mathrm{HM} q^{+}$belongs to the adjoint representation of the gauge group and interacts with the gauge multiplet through the usual minimal coupling:

$$
\nabla^{++} q^{+}=D^{++} q^{+}+i g\left[V^{++}, q^{+}\right]=0 .
$$

The harmonic gauge connection $V^{++}$serves as the prepotential of the $\mathcal{N}=2$ SYM theory. The derivatives $D_{\alpha, \dot{\alpha}}^{+}$do not need a connection in the frame where G-analyticity is manifest (see (6)). The connections for the conjugate harmonic derivative $\nabla^{--}=D^{--}+$ $i g V^{--}$and for the spinor derivatives $\nabla_{\alpha, \dot{\alpha}}^{-}=D_{\alpha, \dot{\alpha}}^{-}+i g A_{\alpha, \dot{\alpha}}^{-}$are determined through the conventional constraints

$$
\begin{aligned}
& {\left[\nabla^{++}, \nabla^{--}\right]=2 D^{0},} \\
& {\left[\nabla^{--}, D_{\alpha, \dot{\alpha}}^{+}\right]=\nabla_{\alpha, \dot{\alpha}}^{-},}
\end{aligned}
$$

where $D^{0}$ is the $U(1)$ charge operator. Next, the field strength is defined by the relation

$$
\left\{\nabla_{\alpha}^{+}, \nabla_{\beta}^{-}\right\}=2 i g \epsilon_{\alpha \beta} \bar{W}
$$

It is covariantly chiral,

$$
\nabla_{\alpha}^{+} \bar{W}=\nabla_{\alpha}^{-} \bar{W}=0
$$

and harmonic independent,

$$
\nabla^{++} \bar{W}=0
$$

as a consequence of the above constraints.

In the free case the curvature $\bar{W}$ satisfies the field equation

$$
\bar{D}_{\dot{\alpha}}^{+} \bar{D}^{+\dot{\alpha}} \bar{W}=0
$$

The coupling to $\mathcal{N}=2 \mathrm{HM}$ matter modifies this equation into

$$
\bar{D}_{\dot{\alpha}}^{+} \bar{D}^{+\dot{\alpha}} \bar{W}=8 i g\left[\tilde{q}^{+}, q^{+}\right],
$$

where $\tilde{q}^{+}$denotes a special conjugation on $S^{2}$ which preserves G-analyticity.

Eqs. (8) and (15) are equivalent to the $\mathcal{N}=4 \mathrm{SYM}$ field equations written down in terms of $\mathcal{N}=2$ superfields. They can be obtained from an off-shell action with manifest $\mathcal{N}=2$ supersymmetry, which allows a straightforward quantization. 


\section{The Konishi multiplet and its 1/2 BPS descendant}

The $\mathcal{N}=2$ Konishi superfield is defined as follows:

$$
K=\operatorname{Tr}\left(\frac{1}{2} \bar{W} W+\tilde{q}^{+} \nabla^{--} q^{+}-q^{+} \nabla^{--} \tilde{q}^{+}\right)=\operatorname{Tr}\left(\bar{\phi} \phi+\bar{q}^{i} q_{i}\right)+\theta \text { terms } .
$$

Using the constraints (1) , (10), (11), (12) and the field equations (8), (15) it is easy to check that

$$
D^{+\alpha} D_{\alpha}^{+} K=12 i g \operatorname{Tr}\left(\bar{W}\left[\tilde{q}^{+}, q^{+}\right]\right)
$$

and

$$
D^{++} K=0 \text {. }
$$

Equation (17) encodes the classical non-conservation of the Konishi current $K=\ldots+$ $\theta^{i} \sigma^{\mu} \bar{\theta}_{i} k_{\mu}(x)+\ldots$ The $\mathrm{H}$-analyticity condition (18) means that the chargeless superfield $K$ is harmonic independent ${ }^{3}$

The quantum corrections lead to an anomaly [9] in the right-hand side of eq. (17):

$$
D^{+\alpha} D_{\alpha}^{+} K=12 i g \operatorname{Tr}\left(\bar{W}\left[\tilde{q}^{+}, q^{+}\right]\right)+c g^{2} \bar{D}_{\dot{\alpha}}^{+} \bar{D}^{+\alpha} \operatorname{Tr}(\bar{W} \bar{W}),
$$

where $c$ is a number. We can simplify eq. (19) by hitting it with $\bar{D}_{\dot{\alpha}}^{+} \bar{D}^{+\dot{\alpha}}$. The quantum anomaly term drops out; in the classical term the derivatives see only $\bar{W}$ and we can use the field equation (15). The result is

$$
\begin{aligned}
\left(D^{+}\right)^{4} K \equiv \bar{D}_{\dot{\alpha}}^{+} \bar{D}^{+\dot{\alpha}} D^{+\alpha} D_{\alpha}^{+} K & =-96 g^{2} \operatorname{Tr}\left(\left[\tilde{q}^{+}, q^{+}\right]\left[\tilde{q}^{+}, q^{+}\right]\right) \\
& =-192 g^{2}\left\{\operatorname{Tr}\left(\tilde{q}^{+} q^{+} \tilde{q}^{+} q^{+}\right)-\operatorname{Tr}\left(\tilde{q}^{+} \tilde{q}^{+} q^{+} q^{+}\right)\right\} .
\end{aligned}
$$

The left-hand side of this equations is annihilated by all of the G-analytic derivatives $D^{+}, \bar{D}^{+}$, and so is the right-hand side, due to the G-analyticity of the HMs. Let us define the single-trace composite operator of $U(1)$ charge +4 (equal to its naive dimension)

$$
\mathcal{K}^{+4}=\operatorname{Tr}\left(\left[\tilde{q}^{+}, q^{+}\right]\left[\tilde{q}^{+}, q^{+}\right]\right) .
$$

It is manifestly G-analytic and in addition is also $\mathrm{H}$-analytic (see eq. (50):

$$
D_{\alpha}^{+} \mathcal{K}^{+4}=\bar{D}_{\dot{\alpha}}^{+} \mathcal{K}^{+4}=D^{++} \mathcal{K}^{+4}=0
$$

Note the appearance of commutators under the trace in (21), which indicates that the operator has been derived through the use of the filed equation (15).

A very similar operator, also both $\mathrm{G}$ - and $\mathrm{H}$-analytic, is obtained by taking the sum in the second line of eq. (20): :

$$
\mathcal{O}^{+4}=\operatorname{Tr}\left(\tilde{q}^{+} q^{+} \tilde{q}^{+} q^{+}\right)+\operatorname{Tr}\left(\tilde{q}^{+} \tilde{q}^{+} q^{+} q^{+}\right) .
$$

\footnotetext{
${ }^{3}$ Note that the similar operator $J=\operatorname{Tr}\left(-\bar{W} W+\tilde{q}^{+} \nabla^{--} q^{+}-q^{+} \nabla^{--} \tilde{q}^{+}\right)$corresponds to one of the $\mathcal{N}=2$ supercurrent multiplets and satisfies the conservation conditions $D^{+\alpha} D_{\alpha}^{+} J=D^{++} J=0$ even in the presence of interaction.

${ }^{4}$ There exist two other quadrilinear G- and H-analytic HM composites, $\operatorname{Tr}\left(\tilde{q}^{+} q^{+} q^{+} q^{+}\right)$and $\operatorname{Tr}\left(q^{+} q^{+} q^{+} q^{+}\right)$, but they are not related to the Konishi multiplet, so we do not consider them here.
} 
Despite the appearance, the two operators $\mathcal{K}^{+4}$ and $\mathcal{O}^{+4}$ are substantially different, but this can only be seen in the interacting quantum theory. In the free case both operators can be viewed as $1 / 2$ BPS operators. In fact, the free Konishi superfield $K_{0}$ of canonical dimension two is a reducible representation of conformal supersymmetry (much like the Konishi current $k^{\mu}(x)$ of canonical dimension three which is a reducible representation of the conformal group). As such, it can be decomposed into several irreducible multiplets (see [4] for details), one of which is the $1 / 2$ BPS projection $\left(D^{+}\right)^{4} K_{0}$. Then the free version of eq. (20) simply means that this $1 / 2$ BPS projection vanishes. This statement is manifestly super-Poincaré invariant because $\left[Q,\left(D^{+}\right)^{4}\right]=0$, but it is also superconformal, since $\left[S,\left(D^{+}\right)^{4}\right] K_{0}=0$ provided that the superfield $K_{0}$ has canonical dimension two. Then, what remains in the free Konishi superfield $K_{0}$ forms a semishort multiplet. As to the right-hand side of eq. (20), in the free case it is not related to the Konishi multiplet. Still, as long as it is made out of free HMs, $\mathcal{K}^{+4}$ undoubtedly is a superconformal primary $1 / 2$ BPS multiplet, just like the other composite $\mathcal{O}^{+4}$.

Now, it is well known that in the quantum interacting theory the Konishi superfield $K$ acquires an anomalous dimension 10, 0, 11. As soon as this happens, the left-hand side of eq. (20) ceased to be superconformally covariant, since $\left[S,\left(D^{+}\right)^{4}\right] K \neq 0$ if $\operatorname{dim} K>2$. In this sense the 1/2 BPS projection $\left(D^{+}\right)^{4} K$ becomes a superconformal descendant of the now long Konishi multiplet $K$. Nevertheless, this $1 / 2$ BPS projection is still conformal primary in the following sense: It can be checked that although the differential operator $\left(D^{+}\right)^{4}$ does not commute with the conformal boost generators $K^{\mu},\left[K^{\mu},\left(D^{+}\right)^{4}\right] \propto \bar{D}^{+} \sigma^{\mu} D^{+} D^{++}$, when applied to the Konishi superfield this commutator still vanishes because of the H-analyticity (or $S U(2)$ irreducibility) condition (18). The above discussion applies to the quadrilinear composite operator $\mathcal{K}^{+4}$ as well, since it is related to the $1 / 2$ BPS non-covariant projection of $K$ through the field equation $(20)$. We conclude that in the interacting quantum theory $\mathcal{K}^{+4}$ should be regarded as a superconformal descendant (but at the same time conformal primary) of the long Konishi multiplet.

On the other hand, there is no obvious reason why the second 1/2 BPS operator $\mathcal{O}^{+4}$ should change its nature when the interaction is switched on. It does not seem to be related via the field equations to any other, long multiplet, so it is likely to remain superconformal primary. If this is true, its dimension should be protected by superconformal symmetry. However, our present understanding of the making of composite operators does not give us a direct and simple test for "primarity". The (non)preservation of this property in the quantum theory is somehow related to the potentially singular nature of the composite operators. It is an interesting problem to find out what exactly "goes wrong" with $\mathcal{K}^{+4}$ but not with $\mathcal{O}^{+4}$. The best we can do at present is to make perturbative tests, see the next section.

Note that in a similar manner we can construct $1 / 2$ BPS operators of lower dimension (charge). These are bilinear or trilinear in the elementary HMs constituents:

$$
\mathcal{O}_{1}^{+2}=\operatorname{Tr}\left(q^{+} q^{+}\right), \quad \mathcal{O}_{2}^{+2}=\operatorname{Tr}\left(\tilde{q}^{+} q^{+}\right), \quad \mathcal{O}_{1}^{+3}=\operatorname{Tr}\left(q^{+} q^{+} q^{+}\right), \quad \mathcal{O}_{2}^{+3}=\operatorname{Tr}\left(\tilde{q}^{+} q^{+} q^{+}\right)
$$

and conjugates. At this low dimension there can be no commutators under the trace, therefore we can expect that the above operators are primary and thus protected. In fact, the bilinears $\mathcal{O}^{+2}$ are current multiplets, they contain various components of the $\mathrm{R}$ symmetry current of the $\mathcal{N}=4$ theory. This gives an additional reason why they should 
be protected.

Another remark concerns the clear difference in the structure of the descendant $\mathcal{K}^{+4}$ and its "parent" superfield $K(16)$. The latter is bilinear in the matter superfields $q^{+}$, but non-polynomial in the gauge superfield. H However, its descendant $\mathcal{K}^{+4}$ is polynomial in the HMs and does not involve any gauge superfields. In this respect it strongly resembles the (supposedly) primary operator $\mathcal{O}^{+4}(23)$. We believe that the much simpler structure of $\mathcal{K}^{+4}$ makes it a better candidate for further investigations than its parent $K$.

\section{BPS operators in perturbation theory}

As discussed in the preceding section, the quadrilinear composites $\mathcal{K}^{+4}$ and $\mathcal{O}^{+4}$, although having the same properties of $1 / 2$ BPS operators in the free case, are expected to behave quite differently in the (perturbative) quantum theory. To get a better feeling of how this happens, it is useful to reexamine the two-point functions of these operators at one loop. Below we outline this calculation for $\mathcal{K}^{+4}$ in the $\mathcal{N}=2$ harmonic superspace formalism [8].

To start with, Wick contractions with the HM Euclidean propagator $\left\langle\tilde{q}^{+}(1) q^{+}(2)\right\rangle_{\theta=0}=$ (12) $/ 4 \pi^{2} x_{12}^{2}$ produce the free two-point function

$$
\left\langle\mathcal{K}^{+4}\left(x_{1}\right) \mathcal{K}^{+4}\left(x_{2}\right)\right\rangle_{\theta=0}=\frac{12 N^{3}\left(N^{2}-1\right)}{\left(4 \pi^{2}\right)^{4}} \frac{(12)^{4}}{x_{12}^{8}},
$$

where $(12) \equiv u_{1}^{+i} u_{2 i}^{+}$is the $S U(2)$ invariant contraction of the harmonic variables at the two points. Here and below we consider only the lowest component of $\mathcal{K}^{+4}$ putting thereby $\theta_{1}=\theta_{2}=0$.

At order $g^{2}$ we have graphs of two types, $J_{1}$ and $J_{2}$ (see Fig. 1). The graphs of the type $J_{1}$ are obtained by inserting a gluon propagator between any two (solid) HM lines. The graphs of the type $J_{2}$ always contain two HM loops connected by a gluon exchange.

\footnotetext{
${ }^{5}$ In the harmonic formulation the non-linear object is the connection $V^{--}$whose expression in terms of the prepotential $V^{++}$via the constraint (9) is non-polynomial [8]; in the more familiar $\mathcal{N}=1$ formulation the non-polynomial dependence is due to the gauge factor in $\operatorname{Tr} \Phi e^{i g V} \Phi$.

${ }^{6}$ The color combinatorics is performed by using the relations $\operatorname{Tr}\left(T^{a} T^{b}\right)=\delta^{a b}, f_{a c d} f_{b c d}=2 N \delta_{a b}$, $f_{a m n} f_{b n p} f_{c p m}=N f_{a b c}$, where $T^{a}$ with $a=1, \ldots N^{2}-1$ are the $S U(N)$ Lie algebra generators.

${ }^{7}$ The self-energy contribution to the HM propagator vanishes in the $\mathcal{N}=2$ formalism.
} 


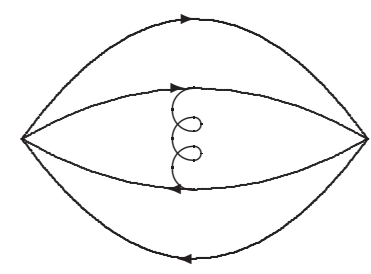

$J_{1}$

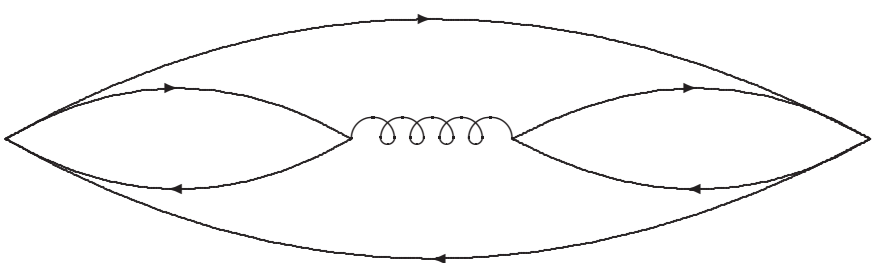

$J_{2}$

Figure 1. Two different topologies contributing to the two-point function of $\mathcal{K}^{+4}$. Only the second graph is responsible for the anomalous dimension.

Explicitly, $J_{1}$ and $J_{2}$ are given by the following analytic superspace integrals:

$$
J_{1} \sim \frac{(12)^{2}}{x_{12}^{4}} \int d u_{5,6} d^{4} \theta_{5,6}^{+} d^{4} x_{5,6} \frac{(15)(52)}{\hat{x}_{15}^{2} \hat{x}_{52}^{2}} \frac{(16)(62)}{\hat{x}_{16}^{2} \hat{x}_{62}^{2}}\left\langle V^{++}(5) V^{++}(6)\right\rangle
$$

and

$$
J_{2} \sim \frac{(12)^{2}}{x_{12}^{4}} \int d u_{5,6} d^{4} \theta_{5,6}^{+} d^{4} x_{5,6} \frac{(15)(51)}{\hat{x}_{15}^{2} \hat{x}_{51}^{2}} \frac{(26)(62)}{\hat{x}_{26}^{2} \hat{x}_{62}^{2}}\left\langle V^{++}(5) V^{++}(6)\right\rangle
$$

Here

$$
\left\langle V^{++}(5) V^{++}(6)\right\rangle=\frac{\left(\theta_{5}^{+}-\left(56^{-}\right) \theta_{6}^{+}\right)^{4}}{2 \pi^{2} x_{56}^{2}} \delta^{(-2,2)}\left(u_{5}, u_{6}\right)
$$

is the propagator of the $\mathcal{N}=2$ prepotential $V^{++}$in the Feynman gauge. With all external $\theta$ 's set to zero, the superinvariant coordinate difference entering the HM propagator between points, e.g. 1 and 5 is defined as follows:

$$
\hat{x}_{15}^{2}=\left(x_{15}-2 i \frac{\left(15^{-}\right)}{(15)} \theta_{5}^{+} \sigma \theta^{+}{ }_{5}\right)^{2}=\hat{x}_{51}^{2},
$$

where $\left(15^{-}\right)=u_{1}^{+i} u_{5 i}^{-}$. The integration over the internal Grassmann and harmonic variables is very easy and we find for $J_{1}$ and $J_{2}$ the following space-time expressions (dropping the propagator factors):

$$
J_{1} \sim \square_{1} \int \frac{d x_{5} d x_{6}}{x_{15}^{2} x_{16}^{2} x_{56}^{2} x_{25}^{2} x_{26}^{2}}, \quad J_{2} \sim \int \frac{d x_{5}}{x_{15}^{4} x_{25}^{4}} .
$$

The integral in $J_{1}$ is finite, therefore conformal invariance tells us that it is proportional to $1 / x_{12}^{2}$. Thus $J_{1}$ contributes only a contact term to the two-point function which vanishes for separated points. The integral $J_{2}$ is logarithmically divergent and computing it, for instance in dimensional regularization, one finds a simple pole, $J_{2} \sim 1 / \epsilon$. The residue determines the anomalous dimension. Performing a multiplicative renormalization and doing carefully 
the color combinatorics, we find for the renormalized operator $\mathcal{K}^{+4}$ the one-loop anomalous dimension $\gamma=3 g^{2} N / 4 \pi^{2}$, i.e. precisely the same as for the renormalized Konishi operator.

This result is in agreement with the discussion in Section 3, were $\mathcal{K}^{+4}$ was shown to arise as a superconformal descendant of the Konishi multiplet. We clearly see that the $1 / 2$ BPS operator $\mathcal{K}^{+4}$, which was a superconformal primary in the free theory, looses this property when the quantum corrections are turned on. Had it remained primary, its dimension would have been protected by the superconformal symmetry.

In fact, this is what happens to the operator $\mathcal{O}^{+4}$. Repeating our one-loop calculation for the two-point function of $\mathcal{O}^{+4}$, this time we find that only the graph $J_{1}$ contributes while $J_{2}$ is absent due to the mismatch between the color antisymmetrization at the interaction vertices and the symmetrization at the external points. However, as we have just seen, the contribution of $J_{1}$ is a contact term, so the two-point function of $\mathcal{O}^{+4}$ does not receive any quantum correction at one loop (for non-coincident points).

Finally, applying the same arguments to the mixed two-point function $\left\langle\mathcal{K}^{+4} \mathcal{O}^{+4}\right\rangle$, we again find that the graph $J_{2}$ cannot contribute, whereas $J_{1}$ still gives a contact term. Thus, the two operators do not mix, at least at one loop. This situation is different from the example in Ref. [6] concerning two dimension four operators in the $\underline{84}$ of $S U(4)$. One of them is a single-trace descendant, but the other is double-trace and they mix at one loop.

The above perturbative test gives indirect evidence that the operator $\mathcal{O}^{+4}$ remains superconformal primary in the quantum theory, while $\mathcal{K}^{+4}$ changes its nature. It would be preferable to have another, direct way of testing "primarity".

Clearly, the descendant $\mathcal{K}^{+4}$ has the same anomalous dimension as its parent operator. Therefore, from the practical point of view, it might be easier to study the quantum properties of $K$ by instead examining $\mathcal{K}^{+4}$. Indeed, $\mathcal{K}^{+4}$ is an analytic gauge-invariant composite which, contrary to $K$, does not involve any covariantizing gauge connection in its definition. Moreover, the existence of a protected superconformal operator with the same quantum numbers and with a very similar structure suggests important simplifications. It is conceivable that those graphs which appear in the perturbative expansion of the two-point functions for both $\mathcal{O}^{+4}$ and $\mathcal{K}^{+4}$ should have a vanishing (or rather, contact term) contribution, as we have just seen in the simplest one-loop example. If this is true, a large subset of graphs could be eliminated from the calculation of the anomalous dimension of $\mathcal{K}^{+4}$. This point, however, requires further study because at higher loops the protection mechanism for $\mathcal{O}^{+4}$ might be more involved, perhaps not due to the convergence of individual graphs but rather to non-trivial color combinatorics leading to cancellation of individual divergences. In any case, the question of understanding how an operator like $\mathcal{O}^{+4}$ might be protected to all orders in perturbation theory is still open and certainly very interesting.

\section{Conclusions}

In this note, in the context of $\mathcal{N}=2$ SCFT we have demonstrated the different perturbative behavior of operators having the same quantum numbers of a 1/2 BPS state. We considered the simplest example of two analytic superfields composed of four HMs. Both of them are superconformal primary operators in the free theory but when the quantum effects

\footnotetext{
${ }^{8}$ This result is stronger than just saying that the operator $\mathcal{O}^{+4}$ has a protected dimension. In fact, this is what is believed to happen to all $1 / 2$ BPS primary operators [12].
} 
are turned on, the first one ceased to be primary with respect to the special conformal supersymmetry and becomes a member of the long unprotected Konishi multiplet. The second one remains a superconformal primary at the quantum level and hence is protected.

As argued in Ref. [4, in some special cases one is able to tell that a short or a semishort operator with given quantum numbers cannot possibly be contained as a descendant in another, long multiplet, and should thus be automatically protected. Our examples are clearly not of this type. At present, we do not have a better way to decide whether one or the other of our 1/2 BPS operators could acquire an anomalous dimension but making perturbative tests. In this context we remark that the various "non-renormalization theorems" for short operators in the literature have always been based on the (implicit) assumption of "primarity". As we have tried to show in this note, demonstrating the "primarity" of a given composite short operator at the quantum level seems equally difficult, in practice equivalent to proving that it is protected. It would be highly desirable to find some intrinsic criterion for determining the superconformal properties of an operator in perturbation theory.

The discussion above raises the intriguing question about what precisely is responsible for the loss of "primarity" of certain operators. Progress in this direction might lead to explaining the mechanism which protects short operators, on the one hand. On the other, it may prove helpful in revealing the deep origin of the anomalous dimension of long multiplets and eventually to its exact evaluation.

Our discussion also applies to the so-called BMN operators recently introduced in the context of the SYM/plane-wave correspondence [13]. Indeed, a BMN operator with impurities is a mixture of operators in different $S U(4)$ representations. It contains, in particular, irreducible $S U(4)$ pieces which are single-trace operators involving commutators of elementary fields. Thus, some of them might be superconformal descendants of lower-dimensional operators. Since the span of dimensions in a supermultiplet is finite, it is conceivable that in the BMN limit of infinite quantum numbers the difference between a primary field and its supersymmetry descendant actually disappears or becomes insignificant. It would be interesting to better understand this point.

\section{Acknowledgements}

We are grateful to C. Callan, S. Ferrara, P. Howe, H. Osborn and E. Sezgin for many useful discussion. G. A. was supported by the DFG and by the European Commission RTN programme HPRN-CT-2000-00131, and in part by RFBI grant N99-01-00166. 


\section{References}

[1] V. K. Dobrev and V. B. Petkova, "All Positive Energy Unitary Irreducible Representations Of Extended Conformal Supersymmetry," Phys. Lett. B 162 (1985) 127.

P. Heslop and P. S. Howe, "On harmonic superspaces and superconformal fields in four dimensions," Class. Quant. Grav. 17 (2000) 3743, hep-th/0005135.

S. Ferrara and E. Sokatchev, "Superconformalinterpretation of BPS states in AdS geometries," Int. J. Theor. Phys. 40 (2001) 935, hep-th/0005151.

[2] E. Witten, "Anti-de Sitter space and holography," Adv. Theor. Math. Phys. 2 (1998) 253, hep-th/9802150.

[3] S. Ferrara and A. Zaffaroni, "Bulk gauge fields in AdS supergravity and supersingletons," hepth/9807090.

L. Andrianopoli, S. Ferrara, E. Sokatchev and B. Zupnik, "Shortening of primary operators in N-extended SCFT(4) and harmonic-superspace analyticity," Adv. Theor. Math. Phys. 3 (1999) 1149, hep-th/9912007.

[4] F. A. Dolan and H. Osborn, "On Short and Semi-Short Representations for Four Dimensional Superconformal Symmetry," hep-th/0209056.

[5] M. Bianchi, S. Kovacs, G. Rossi and Y. S. Stanev, "On the logarithmic behavior in N = 4 SYM theory," JHEP 9908 (1999) 020, hep-th/9906188; "Properties of the Konishi multiplet in N = 4 SYM theory," JHEP 0105 (2001) 042, hep-th/0104016.

[6] A. V. Ryzhov, "Quarter BPS operators in N = 4 SYM," JHEP 0111 (2001) 046, hepth/0109064; E. D'Hoker and A. V. Ryzhov, "Three-point functions of quarter BPS operators in N = 4 SYM," JHEP 0202 (2002) 047, hep-th/0109065.

[7] E. D'Hoker, D. Z. Freedman and W. Skiba, "Field theory tests for correlators in the AdS/CFT correspondence," Phys. Rev. D 59 (1999) 045008, hep-th/9807098.

S. Penati, A. Santambrogio and D. Zanon, "Two-point functions of chiral operators in N = 4 SYM at order g**4," JHEP 9912 (1999) 006, hep-th/9910197; "More on correlators and contact terms in N = 4 SYM at order $\mathrm{g}^{* *} 4$," Nucl. Phys. B 593 (2001) 651, hep-th/0005223.

[8] A. Galperin, E. Ivanov, S. Kalitsyn, V. Ogievetsky and E. Sokatchev, "Unconstrained N=2 Matter, Yang-Mills And Supergravity Theories In Harmonic Superspace," Class. Quant. Grav. 1 (1984) 469.

A. S. Galperin, E. A. Ivanov, V. I. Ogievetsky and E. S. Sokatchev, "Harmonic Superspace," Cambridge Univ. Press (2001) 306 p.

[9] K. Konishi, "Anomalous supersymmetry transformation of some composite operators in SQCD," Phys. Lett. B135, 5 (1984) 439.

[10] D. Anselmi, "Quantum conformal algebras and closed conformal field theory," Nucl. Phys. B 554 (1999) 415, hep-th/9811149.

[11] G. Arutyunov, S. Frolov and A. Petkou, "Perturbative and instanton corrections to the OPE of CPOs in N = 4 SYM(4)," Nucl. Phys. B 602 (2001) 238, E B 609 (2001) 540, hep-th/0010137.

[12] D. Z. Freedman, S. D. Mathur, A. Matusis and L. Rastelli, 'Correlation functions in the CFT $(d) / \operatorname{AdS}(d+1)$ correspondence," Nucl. Phys. B 546 (1999) 96, hep-th/9804058;

P. S. Howe, E. Sokatchev and P. C. West, "3-point functions in N = 4 Yang-Mills," Phys. Lett. B 444 (1998) 341, hep-th/9808162.

[13] D. Berenstein, J. M. Maldacena and H. Nastase, "Strings in flat space and pp waves from N = 4 super Yang Mills," JHEP 0204 (2002) 013, hep-th/0202021. 